\title{
MicroRNA-30a-3p overexpression improves sepsis-induced cell apoptosis in vitro and in vivo via the PTEN/PI3K/AKT signaling pathway
}

\author{
SHUYING YANG, YONGQIANG WANG, HONGMEI GAO and BING WANG
}

\author{
Intensive Care Unit, Tianjin First Central Hospital, Tianjin 300192, P.R. China
}

Received May 19, 2017; Accepted August 10, 2017

DOI: $10.3892 / \mathrm{etm} .2017 .5644$

\begin{abstract}
The aim of the present study was to explain the mechanism of miR-30a-3p overexpression in sepsis-induced cell apoptosis in vitro and in vivo. For the in vitro cell experiments, H9c2 cells were divided into three groups, including the untreated normal control (NC), lipopolysaccharide (LPS)-treated and miRNA (treated with LPS and transfection with miRNA-30a-3p) groups. The cell proliferation and apoptosis were evaluated by MTT assay and flow cytometry, respectively. The relative protein expression levels were measured by western blot assay. In the in vivo experiment, a sepsis rat model was established by intraperitoneal injection of LPS. Sprague Dawley rats were divided into three groups, including the NC, LPS-injected and miRNA (in which model rats were injected with miR-30a-3p vector at the caudal vein) groups. The myocardial morphology in different groups was observed by hematoxylin and eosin staining. In addition, tissue apoptosis and protein expression levels were evaluated by TUNEL and western blot assay, respectively. The results of cell experiments indicated that the cell proliferation rate was significantly increased and the cell apoptosis rate was significantly downregulated in the miR-30a-3p group compared with the LPS group (both $\mathrm{P}<0.05$ ). The relative protein expression of phosphatase and tensin homolog (PTEN) was markedly decreased in the miRNA group compared with the LPS group, while the levels of phosphoinositide 3-kinase (PI3K) and protein kinase $\mathrm{B}$ (AKT) were significantly increased in the miRNA group (all $\mathrm{P}<0.05$ ). In the in vivo experiments, the myocardial morphology of the miRNA group was improved compared with that of the LPS group. Compared with the LPS group, cell apoptosis in the miRNA group was significantly downregulated $(\mathrm{P}<0.05)$, while the relative protein levels (PTEN, PI3K and AKT) in the tissues were also significantly
\end{abstract}

Correspondence to: Dr Hongmei Gao, Intensive Care Unit, Tianjin First Central Hospital, 24 Fukang Road, Tianjin 300192, P.R. China E-mail: gaohongmei2017@126.com

Key words: sepsis, lipopolysaccharide, microRNA-30a-3p, phosphatase and tensin homolog, phosphoinositide 3-kinase, protein kinase B altered $(\mathrm{P}<0.05)$. In conclusion, miR-30a-3p overexpression may improve the sepsis-induced cell apoptosis in vitro and in vivo via the PTEN/PI3K/AKT signaling pathway.

\section{Introduction}

Sepsis is a systemic inflammatory response with a variety of causes, including trauma, infection, surgery and burns. If treatment and management of sepsis is not timely provided, it may lead to shock, multiple organ dysfunction and even mortality $(1,2)$. Myocardial injury is a common complication of sepsis, which results in a high incidence of organ damage. This injury causes severe arrhythmia, cardiac insufficiency and cardiogenic shock, as well as aggravates the damage of other organs and tissues. Therefore, the prevention and treatment of sepsis-induced myocardial injury is of great clinical significance (3-5).

Phosphatase and tensin homolog (PTEN), a phosphatase homolog deleted on chromosome 10 , is a tumor suppressor gene with phosphatase activity (6). PTEN is one of the most important tumor suppressor genes, and is closely associated with numerous human primary and metastatic tumors (7). Previous studies have demonstrated that PTEN overexpression was involved in suppressing the cell activities by regulation of the phosphoinositide 3-kinase (PI3K)/protein kinase B (AKT) signaling pathway (8-10). On the basis of those previous observations, it is hypothesized that PTEN inhibitor may have an effect in improving cell apoptosis.

microRNA (miRNA or miR) molecules serve an important role in the regulation of cell metabolism $(11,12)$. In addition, miRNAs are closely involved in the pathophysiology of lipopolysaccharide (LPS), as miRNA over-expression or knockdown may suppress LPS induced cell apoptosis. In LPS-induced A549 cell apoptosis, miR15a, miR-15b, miR-203 and miR-135a were significantly increased, while the expression levels of miR-23a, miR-205, miR-199 and miR-497 were significantly decreased (13-15). The downregulation of miR-135a expression was observed to relieve the inhibition of the target protein B-cell lymphoma 2 and reduce the apoptosis (16). In RAW264.7 macrophages, LPS upregulated the expression of Toll-like receptor (TLR)-4 and myeloid differentiation primary response gene 88 , while it downregulated the expression of miR-93, which in turn raised its target protein interleukin-1 receptor-associated kinase 4 expression, 
promoting the occurrence and development of inflammation (17).

miR-30a-3p is abnormally expressed in numerous solid tumor cells, including hepatic cancer, lung cancer and cervical cancer, and is closely associated with cell proliferation and apoptosis (18-20). A low expression miR-30a-3p has been observed in hepatocellular carcinoma, while miR-30a-3p overexpression inhibited the proliferation and induced apoptosis in this tumor (21). Using TargetScan software, it was hypothesized that PTEN may be a potential target of miR-30a-3p, since $\mathrm{miR}-30 \mathrm{a}-3 \mathrm{p}$ may participate in the pathophysiological process of cells by regulating the expression of PTEN.

According to these earlier studies, the current study demonstrated that LPS regulated the occurrence and development of apoptosis by affecting the expression of RNA. Thus, it was inferred that relative microRNA overexpression also have an effect in suppressing the development of apoptosis induced by LPS. In the present study, the effect of miR-30a-3p overexpression on cell apoptosis induced by LPS was investigated in vitro in treated $\mathrm{H} 9 \mathrm{c} 2$ cells, as well as in vivo in a rat model of sepsis.

\section{Materials and methods}

Materials and animals. H9c2 cells were purchased from the American Type Culture Collection (Manassas, VA, USA). LPS and the flow cytometry kit (cat no. 4817-60-K; R\&D Systems, Inc., Minneapolis, MN, USA) were purchased from Sigma-Aldrich (Merck KGaA, Darmstadt, Germany). PTEN (cat no. ab109454) PI3K (cat no. ab86714) AKT (cat no. ab8805) and GAPDH (cat no. ab 8245) antibodies were purchased from Abcam (Cambridge, MA, USA). An In situ Cell Death Detection kit to measure the cell apoptosis by TUNEL method (cat no. 11684817910; Roche Diagnostics, Indianapolis, IN, USA). The MTT kit, protein lysis solution, BCA kit and miR-30a-3p vector were purchased from Beyotime Institute of Biotechnology (Haimen, China). Lipofectamine ${ }^{\circledR}$ 2000 was purchased from Thermo Fisher Scientific, Inc. (Waltham, MA, USA). A total of 27 male 6-weeks-old Sprague Dawley (SD) rats weighing 220.5 \pm 15.6 g; $n=9$ per group) were purchased from the Laboratory Animal Center of Sun Yat-sen University (Guangdong, China) and their access to food and drinking water was ad libitum, while they were kept in an environment with a $12 \mathrm{~h}$ light and dark cycle, temperature of $23 \pm 2^{\circ} \mathrm{C}$ and humidity of $55 \pm 10 \%$. The animal experiments conducted in the present study were approved by the Medical Ethics Committee of the Tianjin First Central Hospital (Tianjin, China).

Cell culture and grouping. The H9c2 cells were cultured in high-glucose Dulbecco's modified Eagle's medium supplemented with $10 \%$ fetal bovine serum in a $37^{\circ} \mathrm{C}$ incubator with $5 \% \mathrm{CO}_{2} . \mathrm{H} 9 \mathrm{c} 2$ cells at the logarithmic growth phase were digested with trypsin and then prepared into a cell suspension in 96-well plates $\left(1 \times 10^{4}\right.$ cells/well). The H9c2 cells were divided into three groups, as follows: Normal control (NC) group, in which cells were treated with the empty vector; LPS group, in which cells were treated with $40 \mu \mathrm{g} / \mathrm{ml} \mathrm{LPS}$; and the miRNA group, in which cells were treated with $40 \mu \mathrm{g} / \mathrm{ml}$ LPS for $24 \mathrm{~h}$ before transfection with $10 \mu \mathrm{l} / \mathrm{ml}$ miR-30a-3p (3'-CGACGUUUG-UAGGCUGACUUUC-5') vector using Lipofectamine ${ }^{\circledR}$ 2000. Subsequent experiments were conducted $48 \mathrm{~h}$ following transfection.

MTT assay. The cells in different groups were cultured for $48 \mathrm{~h}$ and then washed with phosphate-buffered saline (PBS). An MTT assay was conducted to examine the cell proliferation according to the manufacturer's protocol provided in the kit, and the absorbance value was measured at a wavelength of $450 \mathrm{~nm}$. The experiment for each group was performed in triplicate.

Cell apoptosis. The H9c2 cell apoptosis was measured using Annexin V-FITC double staining kit (cat no. AP002, GeneScript Co., Ltd., Nanjing, China) and flow cytometry. Cells at the logarithmic phase were seeded in 6 -well plates $\left(1 \times 10^{5}\right.$ cell/well) and cultured in a $37^{\circ} \mathrm{C}$ incubator with $5 \% \mathrm{CO}_{2}$ for $48 \mathrm{~h}$. According to the manufacturer's protocol, cells were digested with trypsin-free EDTA and harvested by centrifugation at 1,000 x g for $3 \mathrm{~min}$ at $4^{\circ} \mathrm{C}$. Next, the supernatant was removed, and then $1 \mathrm{ml}$ cells were added to the PBS tube. Subsequent to washing the cells in the EP tube twice with PBS, $500 \mu \mathrm{l}$ binding buffer and $10 \mu \mathrm{l}$ Annexin V-FITC were added in the dark and mixed for 15 min at $37^{\circ} \mathrm{C}$. Cell apoptosis was detected by flow cytometry, and the experiment was repeated three times.

Animal groups and sepsis model. The SD rats were divided into three groups, as follows: The NC group in which rats were injected with $0.2 \mathrm{ml}$ saline; the LPS group which were injected with $40 \mathrm{mg} / \mathrm{kg}$ LPS in the abdomen to establish the sepsis model; and the miRNA group, in which sepsis model rats were injected with the $0.2 \mathrm{ml} 10 \mu \mathrm{l} / \mathrm{ml} \mathrm{miR-30a-3p} \mathrm{vector} \mathrm{at} \mathrm{the}$ caudal vein following establishment of the sepsis model. A rat model of sepsis was induced by cecal ligation and puncture, as previously described (19). Briefly, the rats were fasted for $12 \mathrm{~h}$ before surgery and then treated with $2 \%$ sodium pentobarbital intraperitoneal anesthesia, followed by fixing the rat limb on the experimental table and disinfecting the skin and middle abdominal region. An abdominal median incision of $1.0 \mathrm{~cm}$ was performed by dissecting the skin and subcutaneous tissue layer by layer. Next, the linea alba and peritoneum were cut open without damage caused in the abdominal cavity, and then the cecum and surrounding adjacent bowel were identified and exposed. The cecum was gently pulled and placed in a sterile gauze using 3-0 suture ligation of the cecal and mesenteric vessels at a distance of $1.5 \mathrm{~cm}$ at the end of cecum. After ligation, the cecum was punctured with a $20 \mathrm{G}$ sterile needle. The puncture point was selected in an avascular area. The intestinal contents were gently squeezed out to ensure the patency of puncturing. The cecum and other tissues were placed back into the abdominal cavity, and a 4-0 suture was used to close the abdominal incision. At the end of the procedure, all rats were injected with $1 \mathrm{ml}$ saline in the abdomen to supplement the blood volume and returned to normal diet. At 6-12 h following surgery, the rats developed fever (rectal temperature, $>40^{\circ} \mathrm{C}$ ), chills, lack of strength and lack of motion, oliguria and shortness of breath, which indicated that the sepsis model was established successfully.

Hematoxylin and eosin $(H \& E)$ staining. After the rats were sacrificed, the myocardial tissue was removed and placed in $10 \%$ formaldehyde solution for $48 \mathrm{~h}$ at room temperature. 
The tissues were embedded in paraffin, cut into $4 \mathrm{~mm}$-thick sections and dehydrated with gradient ethanol. Sections were then stained with H\&E and assessed with the optical microscope.

TUNEL assay. The left ventricular myocardium of the rats was embedded in paraffin, sectioned, and then subjected to TUNEL assay according to the manufacturer's protocol provided in the In situ Cell Death Detection kit. For each rat, 10 slices were observed under a light microscope (magnification, x200) and 10 fields-of-view were randomly selected for analysis using MICRO-COS-MOS MiVnt images (Shanghai Changfang Optical Instrument Co., Ltd., Shanghai, China). The mean optical density (MOD) of positive cells was determined, and the percentage of positive cells in the cytoplasm and nucleus was calculated to determine the cell apoptosis index (AI), according to the following formula: AI $(\%)=\mathrm{MOD} / \mathrm{AI}$. The percentage of cells x100.

Western blot assay. Following treatment for $48 \mathrm{~h}$, the left ventricle myocardial tissues and cells in the different groups were subjected to western blot assay to measure the relative protein expression levels of PTEN, PI3K and AKT. Briefly, radioimmunoprecipitation assay buffer was added, incubated for $30 \mathrm{sec}$ on ice and then left to stand for $\sim 25 \mathrm{~min}$, followed by centrifugation at $12,000 \mathrm{x}$ g at $4^{\circ} \mathrm{C}$ for $30 \mathrm{~min}$. The protein content of each supernatant was determined by the BCA method. Next, 20 samples were taken from each group, $30 \mu \mathrm{g}$ protein from each sample were first resolved in $12 \%$ SDS-PAGE gel and then transferred onto a nitrocellulose membrane (Bio-Rad, Hercules, CA, USA). The membrane was rinsed with PBS and the non-specific binding sites were blocked in a solution of $5 \%$ non-fat milk in PBST $(0.05 \%$ Tween 20 in PBS) for $2 \mathrm{~h}$ at $37^{\circ} \mathrm{C}$, followed by three washes in PBST. The membrane was first incubated with PTEN $(1: 1,000)$, PI3K $(1: 1,000)$, AKT $(1: 1,000)$ and GAPDH $(1: 1,000)$ antibodies at $-4^{\circ} \mathrm{C}$ overnight, which were purchased from Abcam and then washed in PBST buffer. Following washing with Tris-buffered saline/Tween 20 three times, horse radish peroxidase-goat anti rabbit secondary antibodies (1:2,000; cat no. 136812; Abcam) were added and incubated for at least $1 \mathrm{~h}$ at room temperature. The membrane was washed for a further three times and detected with enhanced chemiluminescene using a Bio-Rad system to visualize and quantify the western blots Bio-rad system (Bio-Rad Laboratories, Inc., Hercules, CA, USA).

Statistical analysis. The SPSS version 17.0 statistical software (SPSS, Inc., Chicago, IL, USA) was used for statistical analysis, and data are expressed as the mean \pm standard deviation. Comparisons between two groups were performed with Student's t-test, while one-way analysis of variance followed by a Tukey's test was used to compare the differences between multiple groups. $\mathrm{P}<0.05$ indicated that a difference was statistically significant.

\section{Results}

Cell proliferation determination by MTT assay. As observed in Fig. 1, LPS significantly reduced the cell proliferation rate

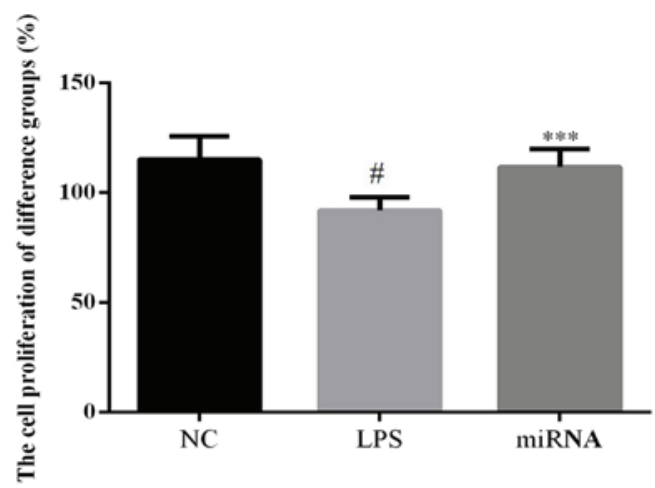

Figure 1. Cell proliferation rate in H9c2 cells, determined by MTT assay. Cells were treated by $40 \mu \mathrm{g} / \mathrm{ml}$ LPS, or by $40 \mu \mathrm{g} / \mathrm{ml}$ LPS followed by miR-30a-3p transfection. ${ }^{\#} \mathrm{P}<0.05$ vs. the NC group; ${ }^{* * *} \mathrm{P}<0.05$ vs. the LPS group. LPS, lipopolysaccharide; miR, microRNA; NC, normal control.

compared with the NC group $(\mathrm{P}<0.05)$. However, the H9c2 cell proliferation rate of the miRNA group was significantly upregulated compared with the LPS group $(\mathrm{P}<0.05)$. This result indicated that miR-30a-3p overexpression improved the LPS-induced suppression of cell proliferation in vitro.

Cell apoptosis determination by flow cytometry. The results of flow cytometry indicated that the cell apoptosis rate of the LPS group was significantly increased compared with NC group and the cell apoptosis rate of the miRNA group was significantly reduced when compared with that in the LPS group (both $\mathrm{P}<0.05$; Fig. 2). This suggested that miR-30a-3p overexpression in $\mathrm{H} 9 \mathrm{c} 2$ cells improved the LPS-induced apoptosis.

Relative protein expression levels of PTEN, PI3K and AKT in cell experiments. As shown in Fig. 3, compared with NC group, the expression of PTEN was significantly increased in the LPS group, while the expression of PI3K and AKT proteins expression were significantly decreased in LPS group (all $\mathrm{P}<0.05$ ) and compared with the LPS group, the PTEN protein expression in the miRNA cell group was significantly downregulated $(\mathrm{P}<0.05)$. By contrast, the PI3K and AKT protein expression levels in the miRNA group were significantly upregulated compared with the LPS group $(\mathrm{P}<0.05$; Fig. 3). Therefore, these findings suggested that miR-30a-3p overexpression may protect against the LPS-induced cell apoptosis by inhibiting PTEN expression and by enhancing the PI3K and AKT protein levels.

$H \& E$ staining of tissues. Myocardial tissues were stained by $\mathrm{H} \& \mathrm{E}$ in the in vivo experiments to examine any alterations caused by sepsis (Fig. 4). In the LPS group, inflammatory cell infiltration was observed, as well as disordered arrangement of myocardial cells and increased interstitial edema. Compared with the LPS group, the inflammatory cell infiltration, myocardial cells arrangement and interstitial edema were significantly improved in in the miRNA group, which meant that the pathological response of the miRNA group was evidently reduced in comparison with that of the LPS group, while it was similar to the NC group (Fig. 4). 

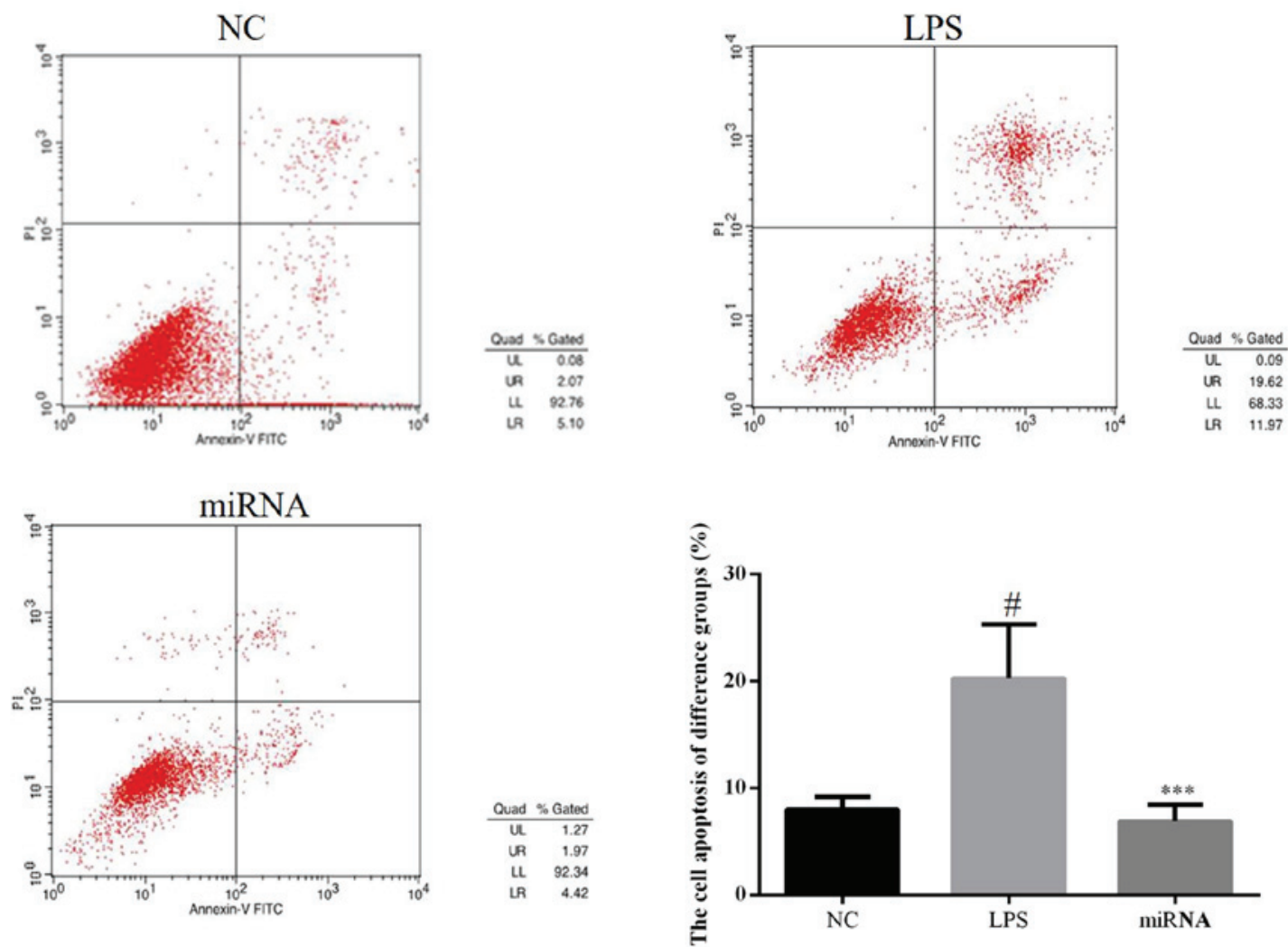

Figure 2. Cell apoptosis in the different groups was determined by flow cytometry. H9c2 cells were treated by $40 \mu \mathrm{g} / \mathrm{ml} \mathrm{LPS}$, or by $40 \mu \mathrm{g} / \mathrm{ml} \mathrm{LPS}$ followed by miR-30a-3p transfection. ${ }^{*} \mathrm{P}<0.05$ vs. the NC group; ${ }^{* * *} \mathrm{P}<0.05$ vs. the LPS group. LPS, lipopolysaccharide; miR, microRNA; NC, normal control.
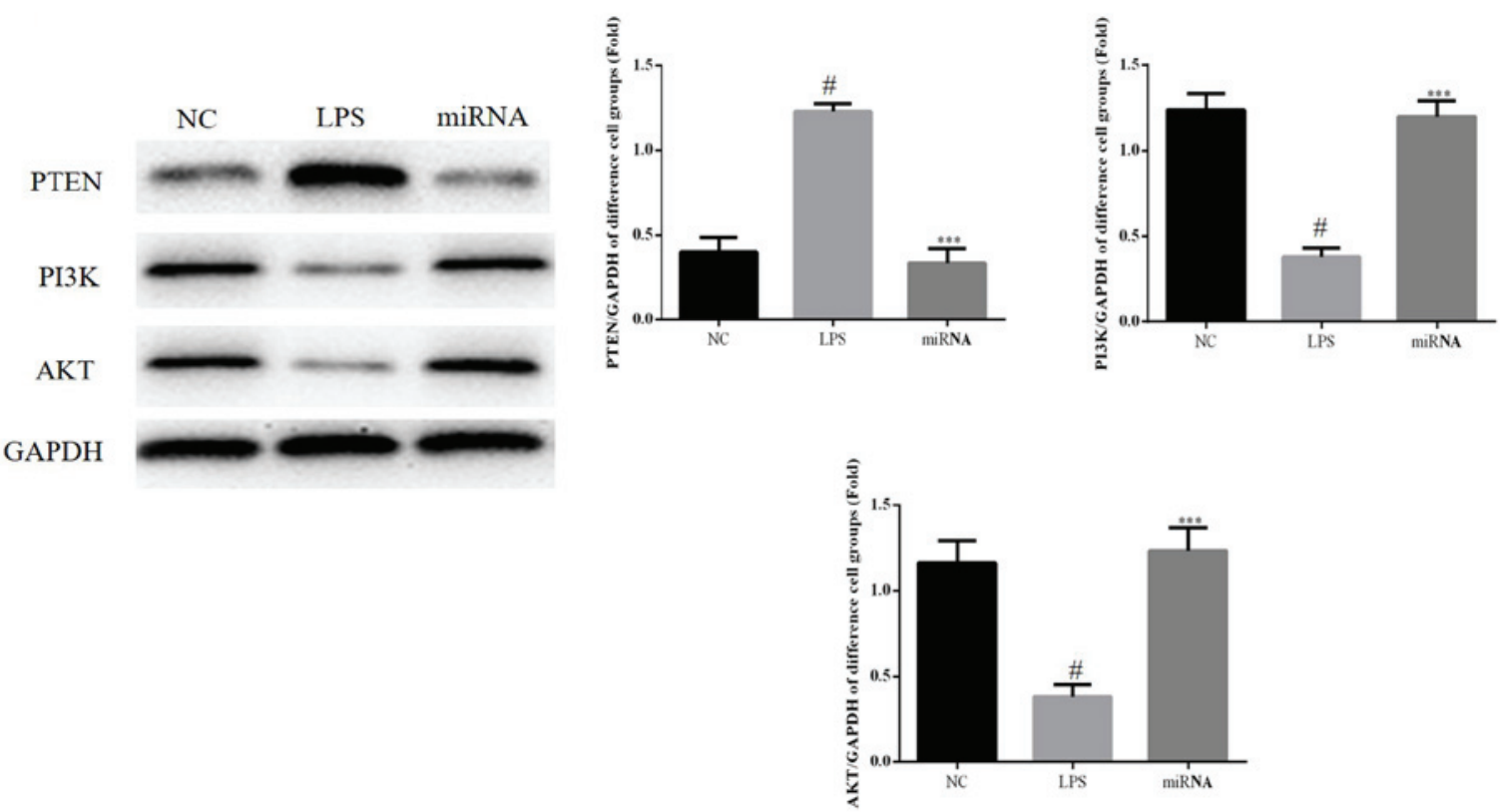

Figure 3. Relative protein expression levels in the in vitro experiments were observed by western blot assay. H9c2 cells were treated by $40 \mu \mathrm{g} / \mathrm{ml} \mathrm{LPS}$, or by $40 \mu \mathrm{g} / \mathrm{ml}$ LPS followed by miR-30a-3p transfection. ${ }^{\prime \prime} \mathrm{P}<0.05$ vs. the NC group; ${ }^{* * *} \mathrm{P}<0.05$ vs. the LPS group. LPS, lipopolysaccharide; miR, microRNA; NC, normal control; PTEN, phosphatase and tensin homolog; PI3K, phosphoinositide 3-kinase; AKT, protein kinase B.

TUNEL assay. TUNEL staining demonstrated that the level of apoptosis in the LPS rat group was significantly higher compared with that in the NC group, and the level of apoptosis in the LPS group was significantly higher compared with that in the miRNA group indicating that miR-30a-3p overexpression prevented the sepsis-induced apoptosis (all $\mathrm{P}<0.05$; Fig. 5).

Relative protein expression levels in rat tissues. The PTEN protein expression of LPS group was significantly increased 


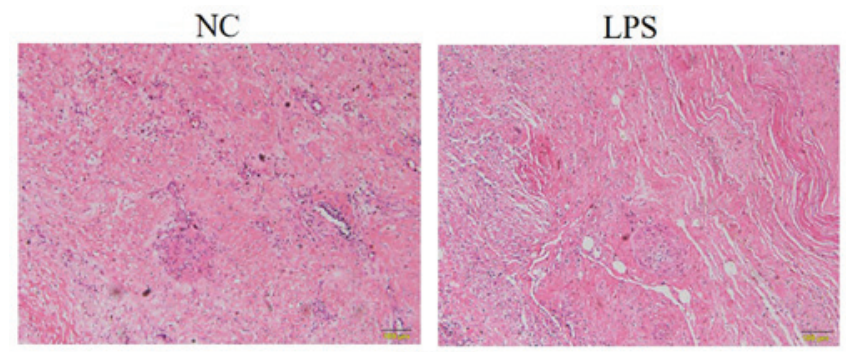

miRNA

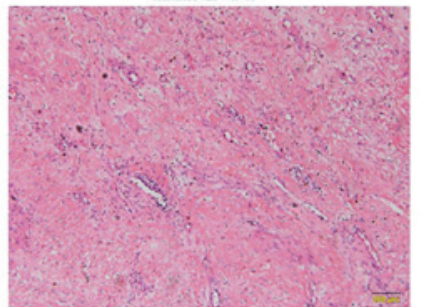

Figure 4. Pathological changes observed in the tissues of different rat groups by hematoxylin and eosin staining (magnification, $\mathrm{x} 200$ ). A rat model of sepsis was established by cecal ligation and puncture (referred to as the LPS group), and model rats were then treated with miR-30a-3p (referred to as the miRNA group). Inflammatory cell infiltration was observed in LPS group, as well as disordered arrangement of myocardial cells and increased interstitial edema. LPS, lipopolysaccharide; miR, microRNA; NC, normal control.

$\mathrm{NC}$
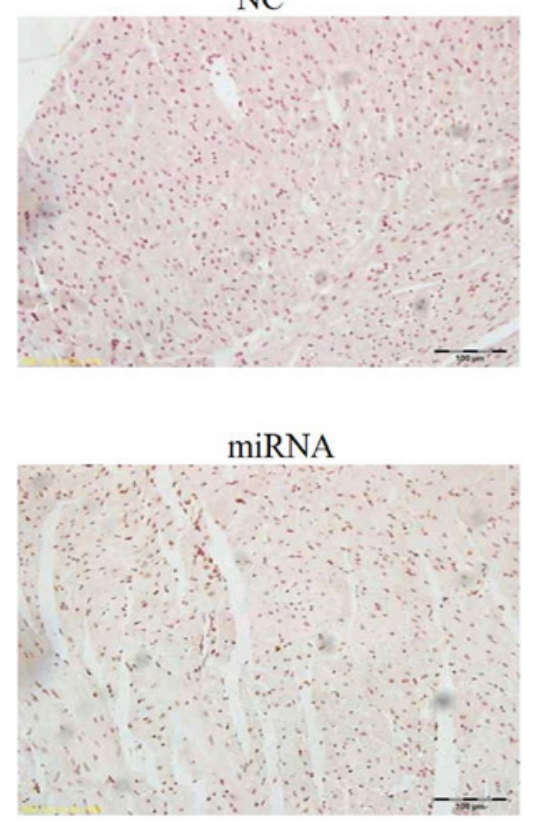

LPS
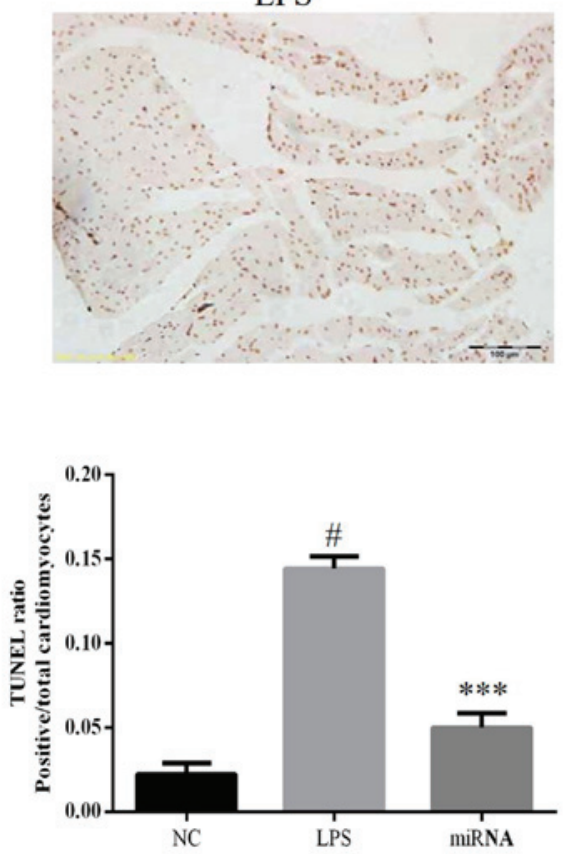

Figure 5. Tissue apoptosis examined by TUNEL assay. A rat model of sepsis was established (referred to as the LPS group), and model rats were then treated with miR-30a-3p (referred to as the miRNA group). ${ }^{\prime} \mathrm{P}<0.05$ vs. the NC group; ${ }^{* * * *} \mathrm{P}<0.05$ vs. the LPS group. LPS, lipopolysaccharide; miR, microRNA; NC, normal control.

while the expression of PI3K and AKT proteins were suppressed compared with the $\mathrm{NC}$ group $(\mathrm{P}<0.05$, respectively). Compared with the LPS group, the PTEN protein expression in the tissues of the miRNA group was significantly downregulated, while the PI3K and AKT protein expression levels in the miRNA group were significantly upregulated (all $\mathrm{P}<0.05$; Fig. 6). These results suggested that miR-30a-3p overexpression may protect against sepsis-induced cell apoptosis by inhibiting PTEN expression and enhancing the PI3K and AKT protein expression levels.

\section{Discussion}

Sepsis is characterized by severe organ dysfunction requiring intensive care and is associated with a high mortality rate (22). The host develops an acute syndrome under exposure to endotoxins, including LPS, which are released from bacterial membranes and trigger a potent inflammatory cytokine response, leading to severe impairment of lipid metabolism (22). LPS binds TLR-4, which leads to the release of proinflammatory cytokines that are essential for a potent 

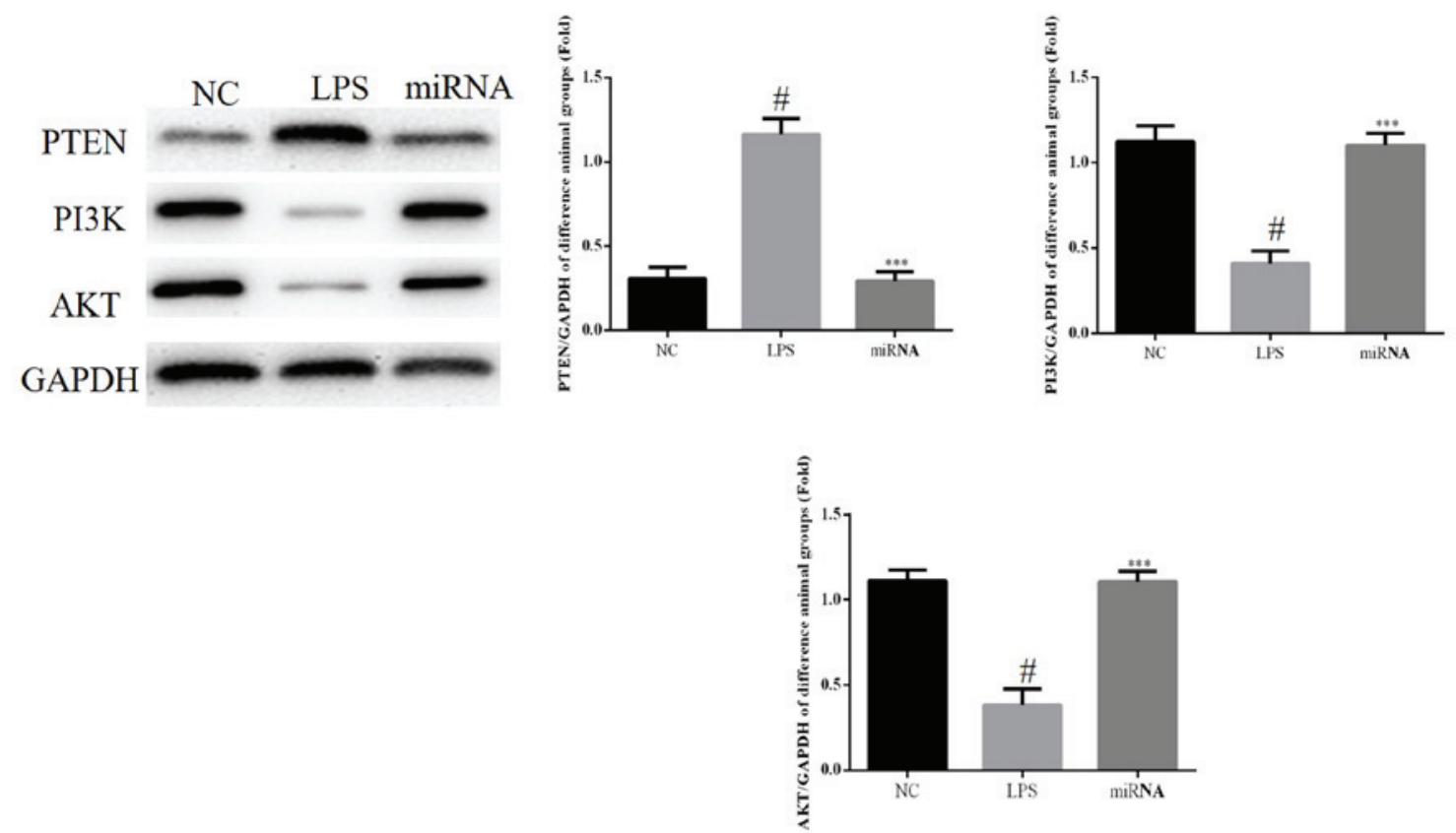

Figure 6. Relative protein expression levels of PTEN, PI3K and AKT in the rat tissues (in vivo experiment). A rat model of sepsis was established (referred to as the LPS group), and model rats were then treated with miR-30a-3p (referred to as the miRNA group). ${ }^{*} \mathrm{P}<0.05$ vs. the NC group; ${ }^{* * * *} \mathrm{P}<0.05$ vs. the LPS group. LPS, lipopolysaccharide; miR, microRNA; NC, normal control; PTEN, phosphatase and tensin homolog; PI3K, phosphoinositide 3-kinase; AKT, protein kinase $\mathrm{B}$.

innate immune response against pathogens (22). The innate immune system is tightly regulated, as excessive inflammation may lead to organ failure and mortality. In the LPS model, septic shock is achieved within a short time (4-6 h) in a simple manner and with a low mortality rate, which is effective for studying the functional changes at the late stage of sepsis shock and its mechanism (23). miRNA is a highly conserved, endogenous non-protein-coding RNA, which serves an important role in maintaining cell growth (20). Previous studies have also demonstrated that miRNAs are involved in inflammation and immune system function $(21,24)$. In epithelial cells, FoxA1 was upregulated by LPS, while miR-17 was significantly downregulated. However, miR-17 mimics significantly inhibited FoxA1 mRNA and protein expression, whereas the miR-17 inhibitor $\mathrm{c}$ significantly increased FoxA1 mRNA and protein expression in LPS-induced type II epithelial cells, which suggested that miRNAs also serve an important role in promoting apoptosis by regulating Fox A1 in the cell experiments (25). miR-375 overexpression was also observed to improve sepsis-induced body injury in a previous study (26). In the present study, the results indicated that miR-30a-3p overexpression improved the cell apoptosis induced by sepsis, as observed in the in vitro and in vivo experiments.

The PTEN gene, which has 9 exons and 8 introns, is located in 10q23.3 and is composed of $200 \mathrm{~kb}$, is the first tumor suppressor gene with a phosphatase activity (27). PTEN encodes a multifunctional protein with a protein and lipid phosphatase activities, which interferes with the PI3K/AKT signal transduction, causing a blockade in the $G$ phase of the cell cycle (28). Furthermore, PTEN has been demonstrated to negatively regulate the PI3K/AKT pathway, as well as induce cell apoptosis and proliferation (29-32).
Previous research has reported that miR modulates PI3K/Akt signaling by directly downregulating PTEN, thereby promoting phosphorylation of Akt, which leads to a decrease in proinflammatory cytokine production, which indicated that miRNAs was associated with PTEN/PI3K/AKT signaling pathway (33). In the present study, the PTEN/PI3K/AKT signaling pathway was evaluated by examining the protein expression by western blot assay in vitro and in vivo. The results confirmed that miR-30a-3p overexpression suppressed the PTEN expression, and enhanced the PI3K and AKT protein expression levels in vitro and in vivo.

The PTEN/PI3K/AKT pathway serves an important role in cell apoptosis. The results of the present study indicated that sepsis induces cardiomyocyte apoptosis by regulation of the PTEN/PI3K/AKT signaling pathway. However, cardiomyocyte cell apoptosis was inhibited with miRNA-30a-3p over-expression in the in vivo and in vitro studies. It was reported that transfection with miR-30a-3p increased the level of PTEN in MG-63 cells, while transfection with miR-30a-3p inhibitor significantly decreased the expression of PTEN in osteosarcoma cells (34).

In conclusion, the in vitro and in vivo results of the present study revealed that $\mathrm{miR}-30 \mathrm{a}-3 \mathrm{p}$ overexpression may have a beneficial effect by improving the LPS-induced or sepsis-induced cell apoptosis via the PTEN/PI3K/AKT pathway. There were however some limitations in the present study, as PTEN was just one target gene of miRNA-30a-3p and it has been unclear whether the miRNA-30a-3p overexpression directly affected the levels of PTEN in LPS- or sepsis-induced cell apoptosis. It was also inferred that miRNA-30a-3p may be a biomarker in sepsis induced myocardial damage. 


\section{References}

1. Camey DE, Matsushima K and Frankel HL: Treatment of sepsis in the surgical intensive careunit. Isr Med Assoc J 13: 694-699, 2011.

2. Kansagara D, Fu R, Freeman M, Wolf F and Helfand M: Intensive insulin therapy in hospitalized patients: A systematic review. Ann Intern Med 154: 268-282, 2011

3. Groening P, Huang Z, La Gamma EF and Levy RJ: Glutamine restores myocardial cytochrome $\mathrm{C}$ oxidase activity and improves cardiac function during experimental sepsis. JPEN J Parenter Enteral Nutr 35: 249-254, 2011.

4. Zhang X, Dong S, Qin Y and Bian X: Protective effect of erythropoietin against myocardial injury in rats with sepsis and its underlying mechanisms. Mol Med Rep 11: 3317-3329, 2015.

5. Zhou X, Lu J, Chen D, Wang W, Cai Q, Li T and Zhang J: Matrix metalloproteinase- 8 inhibitors mitigate sepsis-induced myocardial injury in rats. Chin Med J (Engl) 127: $1530-1535,2014$

6. Li DM and Sun H: TEPI, encoded by a candidate tumor suppressor locus, is a novel protein tyrosine phosphatase regulated by transforming growth factors beta. Cancer Res 57 2124-2129, 1997.

7. Carnero A, Blanco-Aparicio C, Renner O, Link W and Leal JF: The PTEN/PI3K/AKT signaling pathway in cancer, therapeutic implications. Curr Cancer Drug Targets 8: 1568-1570, 2008.

8. Conde-Perez A and Larue L: PTEN and melanomagenesis. Future Oncol 8: 1109-1120, 2012.

9. Akca H, Demiray A, Asian M, Acikbas I and Tokgun O: Tumor suppressor PTEN enhanced enzyme activity of GPx, SOD and caralase by suppression of PI3K/AKT pathway in non-small cell lung cancer cell lines. J Enzyme Inhib Med Chem 28: 539-544, 2013 .

10. Song MS, Salmena L and Pandolfi PP: The functions and regulation of the PTEN tumour suppressor. Nat Rev Mol Cell Biol 13: 283-296, 2012

11. Krol J, Loedige I and Filipowicz W: The widespread regulation of microRNA biogenesis, function and decay. Nat Rev Genet 11: 597-610, 2010

12. Bhaskaran M and Mohan M: MicroRNAs: History, biogenesis, and their evolving role in animal development and disease. Vet Pathol 51: 759-774, 2014

13. Liu S, Liu C, Wang Z, Huang J and Zeng Q: microRNA-23a-5p acts as a potential biomarker for sepsis-induced acute respiratory distress syndrome in early stage. Cell Mol Biol (Noisy-le-grand) 62: 31-37, 2016.

14. Zhou W, Wang J, Li Z, Li J and Sang M: MicroRNA-205-5b inhibits HMGB1 expression in LPS-induced sepsis. Int J Mol Med 38: 312-318, 2016.

15. Liu GD, Zhang H, Wang L, Han Q, Zhou SF and Liu P: Molecular hydrogen regulates the expression of miR-9, miR-21 andmiR-199 in LPS-activated retinal microglia cells. Int J Ophthalmol 6: 280-285, 2013

16. Zhao J, Li X, Zou M, He J, Han Y, Wu D, Yang H and Wu J: miR-135a inhibition protects A549 cells from LPS-induced apoptosis by targeting Bcl-2. Biochem Biophys Res Commun 452: 951-957, 2014

17. Xu Y, Jin H, Yang X, Wang L, Su L, Liu K, Gu Q and Xu X: MicroRNA-93 inhibits inflammatory cytokine production in LPS-stimulated murine macrophages by targeting IRAK4. FEBS Lett 588: 1692-1698, 2014.
18. Wang W, Lin H, Zhou L, Zhu Q, Gao S, Xie H, Liu Z, Xu Z, Wei J, Huang $X$ and Zheng S: MicroRNA-30a-3p inhibits tumor proliferation, invasiveness and metastasis and is downregulated in hepatocellular carcinoma. Eur J Surg Oncol 40: 1586-1594, 2014.

19. Hua S, Liu X, Lv S and Wang Z: Protective effects of Cucurbitacin $\mathrm{B}$ on acute lung injury induced by sepsis in rats. Med Sci Monit 23: 1355-1362, 2017.

20. Croce CM: Causes and consequences of microRNA dysregulation in cancer. Nat Rev Genet 10: 704-714, 2009.

21. Montagner S, Orlandi EM, Merante S and Monticelli S: The role of miRNAs in mast cells and other innate immune cells. Immunol Rev 253: 12-24, 2013.

22. El Kamouni S, El Kebbaj R, Andreoletti P, El Ktaibi A, Rharrassi I, Essamadi A, El Kebbaj MS, Mandard S, Latruffe N, Vamecq J, et al: Protective effect of Argan and olive oils against LPS-induced oxidative stress and inflammation in mice livers. Int J Mol Sci 18: E2181, 2017.

23. Zhang B, Liu C, Yang N and Wang X: A comparative study of changes of autophagy in rat models of CLP versus LPS induced sepsis. Exp Ther Med 14: 2194-2200, 2017.

24. Liston A, Papadopoulou AS, Danso-Abeam D and Dooley J: MicroRNA-29 in te adaptive immune system: Setting the threshold. Cell Mol Life Sci 69: 3533-3541, 2012.

25. Xu Z, Zhang C, Cheng L, Hu M, Tao H and Song L: The microRNA miR-17 regulates lung FoxA1 expression during lipopolysaccharide-induced acute lung injury. Biochem Biophys Res Commun 445: 48-53, 2014.

26. Sheng B, Zhao L, Zang X, Zhen J and Chen W: miR-375 ameliorates sepsis by downregulating miR-21 level via inhibiting JAK2-STAT3 signaling. Biomed Pharmacother 86: 254-261, 2017.

27. Yoshimoto M, Ding K, Sweet JM, Ludkovski O, Trottier G, Song KS, Joshua AM, Fleshner NE, Squire JA and Evans AJ: PTEN losses exhibit heterogeneity in multifocal prostatic adenocarcinoma and are associated with higher Gleason grade. Mod Pathol 26: 435-447, 2013

28. Slomovitz BM and Coleman RL: The PI3K/AKT/mTOR pathway as a therapeutic target in endometrial Cancer. Clin Cancer Res 18: 5856-5864, 2012.

29. Hlobilková A, Knillová J, Bártek J, Lukás J and Kolár Z: The mechanism of action of the tumour suppressor gene PTEN. Biomed Pap Med Fac Univ Palacky Olomouc Czech Repub 147: 19-25, 2003.

30. Selvendiran K, Tong L, Vishwanath S, Bratasz A, Trigg NJ, Kutala VK, Hideg K and Kuppusamy P: EF24 induces G2/M arrest and apoptosis in cisplatin-resistant human ovarian carcer cells by increasing PTEN expression. J Biol Chem 282: 28609-28618, 2007.

31. Wang H, Liu H, Chen K, Xiao J, He K, Zhang J and Xiang G: SIRT1 promotes tumorigenesisol hepatocellular carcinoma through PI3K/TEN/AKT signaling. Oncol Rep 28: 311-318, 2012.

32. Osaki M, Oshimura M and Ho H: PI3K-AKT pathway: Its functions and alterations in human cancer. Apoptosis 9: 667-676, 2004.

33. Kalantari P, Harandi OF, Agarwal S, Rus F, Kurt-Jones EA, Fitzgerald KA, Caffrey DR and Golenbock DT: miR-718 represses proinflammatory cytokine production through targeting phosphatase andtensin homolog (PTEN). J Biol Chem 292: 5634-5644, 2017.

34. Zhong B, Guo S, Zhang W, Zhang C, Wang Y and Zhang C: Bioinformatics prediction of miR-30a targets and its inhibition of cell proliferation of osteosarcoma by up-regulating the expression of PTEN. BMC Med Genomics 10: 64, 2017. 